\title{
Waves from the Shoemaker-Levy 9 impacts
}

\author{
By ANDREW P. INGERSOLL \\ AND HIROO KA NAMORI
}

\begin{abstract}
Division of Geological and Planetary Sciences, California Institute of Technology, Pasadena, CA 91125, USA
\end{abstract}

Images of Jupiter taken by the Hubble Space Telescope (HST) reveal two concentric circular rings surrounding five of the impact sites from comet Shoemaker-Levy 9 (SL9). The rings are visible 1.0 to 2.5 hours after the impacts. The outer ring expands at a constant rate of $450 \mathrm{~m} \mathrm{~s}^{-1}$. The inner ring expands at about half that speed. The rings appear to be waves. Other features (diffuse rings and crescent) further out appear to be debris thrown out by the impact. Sound waves ( $p$-modes), internal gravity waves ( $g$-modes), surface gravity waves ( $f$-modes), and rotational waves ( $r$-modes) all are excited by the impacts. Most of these waves do not match the slow speed, relatively large amplitude, and narrow width of the observed rings. Ingersoll and Kanamori have argued that internal gravity waves trapped in a stable layer within the putative water cloud are the only waves that can match the observations. If they are correct, and if moist convection in the water cloud is producing the stable layer, then the $\mathrm{O} / \mathrm{H}$ ratio on Jupiter is roughly ten times that on the Sun.

\section{Introduction}

Much of what we know about the interior of the Earth has come from the study of seismic waves-a branch of seismology. Recently, much has been learned about the interior of the Sun from helioseismology. Now, the SL9 impacts give us an opportunity to do jovian seismology. The waves probe Jupiter's atmosphere to depths that cannot be reached by remote-sensing instruments. Their speed of propagation reveals properties of the medium through which they are passing. The speed is a definite, measured quantity - perhaps the most well-determined property of the atmosphere to emerge from the SL9 impacts. The interpretation, in terms of composition and structure of Jupiter's atmosphere, depends on knowing what kind of waves were seen and what levels they are sounding.

Waves may be classified by the different restoring forces that cause them to propagate. Sound waves have compressibility as the restoring force-the increase in pressure that accompanies an increase in density. Sound waves are also known as acoustic waves, and when trapped inside a star they are known as $p$-modes. Surface waves have gravity as the restoring force. In astronomy they are known as $f$-modes. The dispersion relation - the relation between frequency and wavelength - is the same for the surface of a star as for the surface of a deep ocean. The density structure of the ambient medium does not enter. Internal gravity waves have buoyancy as the restoring force-the difference between a parcel's own weight and that of the ambient fluid it displaces. Inertia-gravity waves are simply gravity waves modified by rotation, in which case Coriolis forces provide an additional restoring force. In astronomy, these are the $g$ - and $r$-modes. Rossby waves are a special set of $r$-modes that have vortex tube stretching as the primary restoring force: Parcels displaced across topographic contours or across latitude circles develop anomalous vorticity and are sent spinning back. Steady geostrophic flow is a limiting case of Rossby waves. The flow does not cross topographic contours or latitude circles, and the frequency is zero.

Further classification is possible depending on whether one is using ray theory or normal modes to describe the waves. Seismologists have a scheme based on ray theory 
that keeps track of the reflections and transmissions at internal interfaces (e.g., the coremantle boundary) and internal reflections off the surface. The direct ray is a special case because it propagates without reflection, usually from one point on the surface to another point on the surface. Normal mode theory provides an alternate classification. In general, each ray can be viewed as a superposition of normal modes, and vice versa. But special rays like the direct ray, and low-order modes-those with a small number of nodes on the surface and interior of the sphere, generally have very different properties.

Certain modes are trapped within horizontal layers. For example, the sound channel in the ocean is an acoustic waveguide - a layer where the speed of sound is a minimum with respect to depth. Near-horizontal rays are refracted back toward this layer by the gradients in the speed of sound. Normal modes centered on this layer have exponential tails in the high-velocity layers above and below. Jupiter's atmosphere contains a similar waveguide, centered at the tropopause where the temperature and hence the speed of sound is a minimum. The atmosphere may also contain a waveguide for internal gravity waves, which can be trapped within a stably stratified layer (one where entropy per unit mass increases with altitude) if the layers above and below are neutral (entropy per unit mass independent of altitude).

A wave is a propagating disturbance. The speed of propagation is larger than the speed of the fluid. An atmospheric wave becomes visible if a vapor condenses to form a cloud which then evaporates as the wave moves past. Advection, on the other hand, is moving fluid. The advancing front becomes visible if it carries particles with it. Both waves and advection from the SL9 impacts might manifest themselves as expanding circular rings surrounding the impact sites.

Circular wave-like features were seen in Hubble Space Telescope (HST) images (Hammel et al. 1995) and in Earth-based images at $3.42 \mu \mathrm{m}$ in the strong $\nu_{3}$ band of methane (McGregor et al. 1995). During the first few hours, the rings spread out from the impact sites like ripples in a pond. The observations are described in $\S 2$. We also discuss the possibility that the expansion of the rings is due to outward motion (advection) rather than wave propagation. The $p$-modes, $f$-modes, and $r$-modes are discussed in $\S 3$. These wave types do not fit the observations, because they have either the wrong speed of propagation, the wrong waveform, or the wrong amplitude. The only waves that fit are $g$-modes of a special type - those trapped within a stable layer at the level of the jovian water cloud. These waves are discussed in $\S 4$. Fitting to the observations has important implications about the abundance of water on Jupiter. The implications and a comparison of results from the Galileo probe are described in $\S 5$.

\section{Observations}

Hammel et al. (1995) analyzed the atmospheric waves seen in the HST images. Figure 1 is an orthonormal projection of an image taken in a filter at $555 \mathrm{~nm}$ of the $\mathrm{G}$ impact site. A colored version of the same figure appeared in West et al. (1995) and as the cover photo accompanying Ingersoll \& Kanamori (1995). Hammel et al. (1995) show singlefilter images of rings around other impact sites. In Fig. 1, the time interval between the impact and the exposure is $109 \mathrm{~min}$. North is at the top. The radius of the prominent dark ring is $3700 \mathrm{~km}$. A smaller dark ring, of radius $1750 \mathrm{~km}$, is faintly visible. The two rings appear to be concentric, although the smaller ring is partially obscured by the prominent dark spot to the southeast of center. The dark streak outside and to the west of the ring is the site of the D impact, which occurred 20 hours earlier.

Figure 2, which is re-drawn from Hammel et al. (1995), shows measurements of the radii of the circular rings that were seen spreading outward from the impact sites. The 
Figure 1. Hubble Space Telescope image of the G impact site. The image was taken through the $555 \mathrm{~nm}$ filter 109 minutes after the impact, and has been map-projected to show the view from a point directly over the site. The radius of the prominent dark ring is $3700 \mathrm{~km}$.

measurements cover the period from 1 hour to 2.5 hours after the impacts. Five impact sites-A, E, G, Q1, and R-were observed during this time, and the data from the different sites are superposed. Other impact sites were observed one or more jovian rotations later (ten hours or more after the impacts), and no rings were visible.

There are two sets of points in Fig. 2. The upper points lie on a well-defined straight line with slope $450 \mathrm{~m} \mathrm{~s}^{-1}$ and intercept $500 \mathrm{~km}$ (radius $r=500 \mathrm{~km}$ at time $t=0$ ). This is the "fast" wave, whose speed is $450 \mathrm{~m} \mathrm{~s}^{-1}$. The other set of points are clustered near 
Time-Distance Curve (Travel-Time Curve)

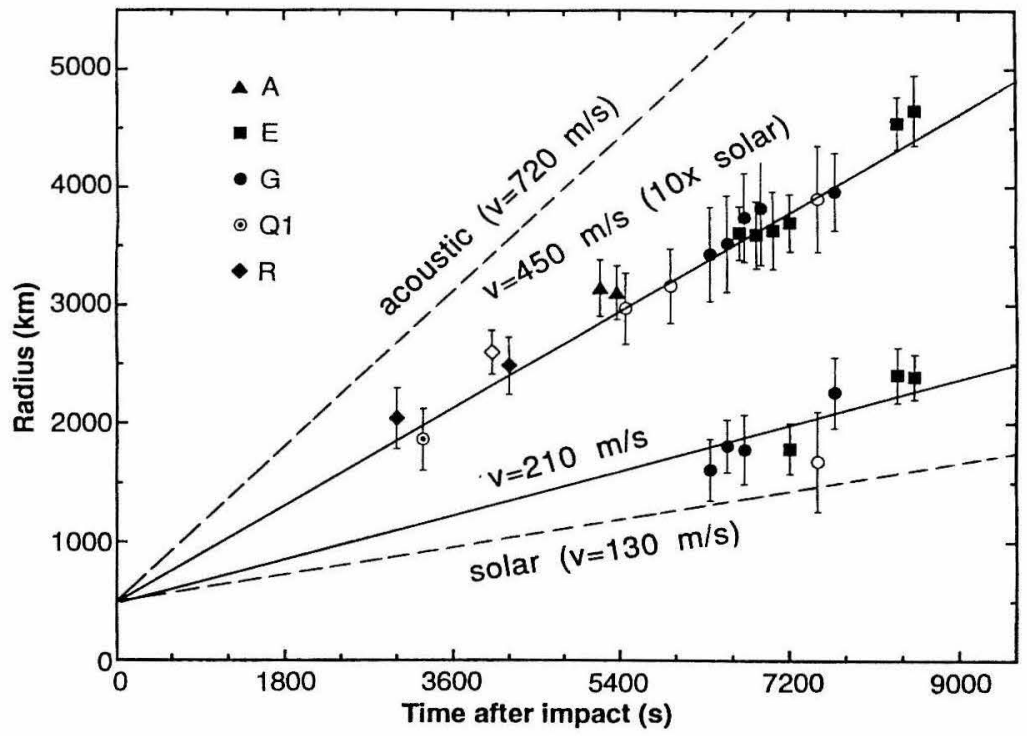

Figure 2. Time-distance curve for the circular rings shown in Fig. 1. The shape of the symbol identifies the fragments A, E, G, Q1, and R. Open symbols identify data taken with the methane $(889 \mathrm{~nm})$ filter. Closed symbols are for all other filters. The intercept was determined from the fit to the upper set of points. "Acoustic" refers to the speed of sound at the temperature minimum. "Solar" and " $10 \times$ solar" refer to the speed of a gravity wave in the water cloud with solar and 10×solar abundance of water. Redrawn from Hammel et al. (1995).

$r=2000 \mathrm{~km}$ and $t=7000 \mathrm{sec}$. These are measurements of a faint inner ring that was seen for impact sites $\mathrm{E}$ and $\mathrm{G}$. The speed is hard to measure. The unconstrained straight line through the lower points has a negative intercept. The line shown was constrained to have the same intercept as the line through the upper points, assuming that both waves started with the same radius, $500 \mathrm{~km}$, at $t=0$. The intercept is thus a measure of the size of the initial disturbance. Note that the constrained line passes through all the error bars. Its slope is $210 \mathrm{~m} \mathrm{~s}^{-1}$, which we take as the speed of the inner ring - the "slow" wave.

The constant velocity suggests that the ring is a propagating wave. The velocity would decrease with time if the particles were carried (advected) with the disturbance. Advection is a non-linear process - the speed of propagation depends on the amplitude. And as the amplitude decays due to geometrical spreading, the speed should decrease. Other evidence of wave-like behavior is that the points from different impacts fall on the same straight line. If material were flowing outward behind a shock or other non-linear disturbance the speed would depend on the energy of impact, and the points would fall on different lines. For a linear wave the speed depends only on properties of the medium.

Zahnle (1995) discusses advection as a possible source of the ring. In an axisymmetric numerical model of the impact, particles that were near the axis before the impact spread out into a flat disk just above the tropopause. The average speed is close to $450 \mathrm{~m} \mathrm{~s}^{-1}$ for the first 1000 seconds, but then the spreading stops. The final radius of the disk is $500 \mathrm{~km}$, which is only $12 \%$ of the maximum radius that was observed (Fig. 2).

Zhang \& Ingersoll (1995) use a similarity solution (Zel'dovich \& Raizer 1967) to reproduce Zahnle's results. The parcel of heated air - the mushroom cloud-rises under its own buoyancy to where its mean entropy matches that of the environment. There, just above the tropopause, the air spreads horizontally, forming a flat disk - a concave 
lens-shaped structure symmetric about a vertical axis. The outer radius $r_{0}$ varies as $t^{n}$ and the speed varies as $t^{n-1}$, where $n$ is a constant to be determined. To conserve volume, the thickness $h$ varies as $t^{-2 n} f\left(r / r_{0}(t)\right)$, where $f$ is a function to be determined. From dimensional analysis or from the Bernoulli and hydrostatic equations, the speed is proportional to $N h_{0}$, where $N$ is the Brunt-Väisälä frequency of the ambient atmosphere and $h_{0}$ is the thickness at the outer edge. Equating powers of $t$ in the two expressions for the speed yields $n=1 / 3$, so that $r_{0} \propto t^{1 / 3}$. Such a power law does not fit the data in Fig. 2, so Zhang \& Ingersoll (1995) conclude that the rings cannot be advection of particles associated with the spreading lens. Advection might occur during the first 1000 seconds when the dark material spreads to a radius of $500 \mathrm{~km}$, but subsequent spreading at constant speed must be due to propagation.

Other features of the $\mathrm{G}$ impact site, including the large diffuse crescent outside the ring, are discussed by Hammel et al. (1995), McGregor et al. (1995), Pankine \& Ingersoll (1995), and others. The outer edge of the crescent is about $13,000 \mathrm{~km}$ from the impact site, which is four times the maximum height of the plumes. Hammel et al. (1995) argue that the crescent is impact debris from the plumes and that the outer edge corresponds to plume material that was launched upward at a $45^{\circ}$ angle with respect to the vertical. One hour after the impacts the crescent is still spreading, but at a slower rate than that implied by the mean radius divided by the time. Zahnle (1995) argues that this spreading is due to sliding of the debris along the top of the atmosphere.

Earth-based imaging in the strong methane band at $3.42 \mu \mathrm{m}$ reveals a large ring outside the crescent (McGregor et al. 1995). The material must be high in the atmospherehigher than the material observed in the HST images. The inner and outer radii of the ring are 15,000 and $18,000 \mathrm{~km}$, respectively. Like the crescent, the ring continues to expand for 2 hours after the impacts, but at a slower rate than that suggested by the mean radius divided by the time. McGregor et al. (1995) discuss two possibilities-that the ring is sliding debris and that it is a propagating wave. The measured expansion velocity modestly exceeds the sound speed in the undisturbed stratosphere, suggesting the possibility of a weak shock wave. Numerical simulations, reviewed by Zahnle (1995), show that sliding does occur. McGregor et al. (1995) regard the large ring as a challenge to our understanding. We shall focus our discussion on the smaller, slower rings that appear in the HST images.

The brown ring material is similar to the impact debris. HST images in the $889 \mathrm{~nm}$ band suggest that it is located in the stratosphere somewhere between the $1 \mathrm{mb}$ and $300 \mathrm{mb}$ levels (Hammel et al. 1995; West et al. 1995). West et al. (1995) argue that the particles are created by condensation and destroyed by evaporation as the wave moves past. This requires that the particles be volatile. West et al. (1995) cite polymerized HCN as an interesting candidate for the composition of the brown material. Zahnle (1995) regards $\mathrm{S}_{8}$ as the most likely candidate, but he is skeptical of all condensates. Finding a volatile material that condenses at just the right temperature and pressure is difficult. From a chemical point of view, it is easier to postulate that the brown material is refractory - either carbon dust or silicate dust from the comet that is advected with the fluid. But then one has to explain why the material forms a narrow ring that spreads at constant speed. We shall proceed under the assumption that the rings represent a propagating wave, even though the composition of the ring material is uncertain. 


\section{Surface waves, Acoustic waves, and Rossby waves}

\section{1. $f$-modes}

The $f$-modes are surface gravity waves. As with all waves considered here the fluid motion is adiabatic, so the entropy of each fluid parcel is constant. For $f$-modes, the density, pressure, temperature, and all other thermodynamic variables are constant as well (Gill 1982; Goldreich \& Kumar 1990). Therefore the $f$-modes can only be detected by measuring displacement, velocity, or acceleration. This implies that the rings seen in the HST images are not $f$-modes, since HST is sensitive mainly to temperature. That is, if the particles are a condensate they are probably responding mostly to the Lagrangian temperature perturbation (that experienced by a parcel) as the wave moves past. For $f$-modes this perturbation is zero, so the wave should be invisible in HST images.

For wavelengths that are short compared to the radius of Jupiter, the speed of propagation (phase speed) of $f$-modes is $\sqrt{g / k}$, where $k$ is the horizontal wavenumber. This dispersion relation is the same as that for waves on the surface of a deep ocean. The type of fluid and its thermodynamic state do not enter, because the perturbations to the state variables are zero. The waves are highly dispersive. Those whose group velocity matches the $450 \mathrm{~m} \mathrm{~s}^{-1}$ speed observed in HST images have a wavelength of order $200 \mathrm{~km}$. The speed is greater for longer wavelengths. Kanamori (1993) computed surface waves as a superposition of $f$-modes excited by momentum transfer from the comet impact.

\section{2. $p$-modes}

Several authors (Deming 1994; Gough 1994; Lognonné et al. 1994) have calculated the shape of the pulse propagating outward from a hypothetical impact site. Figure 3, reprinted from Lognonné et al. (1994), shows the particle displacement over a period of several days at two locations, $90^{\circ}$ and $180^{\circ}$ away from the impact point. From top to bottom, the first and third curves are for $f$-modes only. The other two curves include both $f$-modes and $p$-modes - those waves that propagate into the interior of the planet. The low-frequency $p$-modes are trapped in the interior by the cold surface layers; the high-frequency $p$-modes leak energy into the stratosphere and are dissipated.

For a spherical non-rotating planet, all the waves converge on the antipode (the point $180^{\circ}$ from the impact site), though not at the same time. Lognonne et al. (1994) assume an impact energy of $5 \times 10^{27} \mathrm{erg}$, and obtain displacements of order $100 \mathrm{~m}$ at the antipode and $4 \mathrm{~m}$ at the $90^{\circ}$ point. The multiple packets in Fig. 3 represent waves that have gone multiple times around the planet. Scattering and absorption by jovian winds and turbulence are not included in the calculation. Clearly, the packets shown do not match the waves seen in the HST images. They propagate too fast and they are spread out too far. Except near the antipodes, their amplitudes are likely to be smaller than the normal jovian weather noise.

\subsection{Normal mode oscillations}

Waves that go several times around the planet can be regarded as superpositions of global normal modes (Lee et al. 1994; Mosser et al. 1995). Each $p$-mode has two angular order numbers, which give the number of zero-crossings in latitude and longitude, respectively, and one radial order number. The $f$-modes have only the two angular order numbers. The frequency of the mode is a unique function of these numbers. In principle, the modes with low angular order numbers should be visible from Earth, since Earth-based telescopes can resolve features that are less than 1/50 the diameter of Jupiter. In practice, the amplitude must be large enough to stand out above the fluctuations associated with jovian weather. Such large amplitudes are unlikely, although several searches were made. 

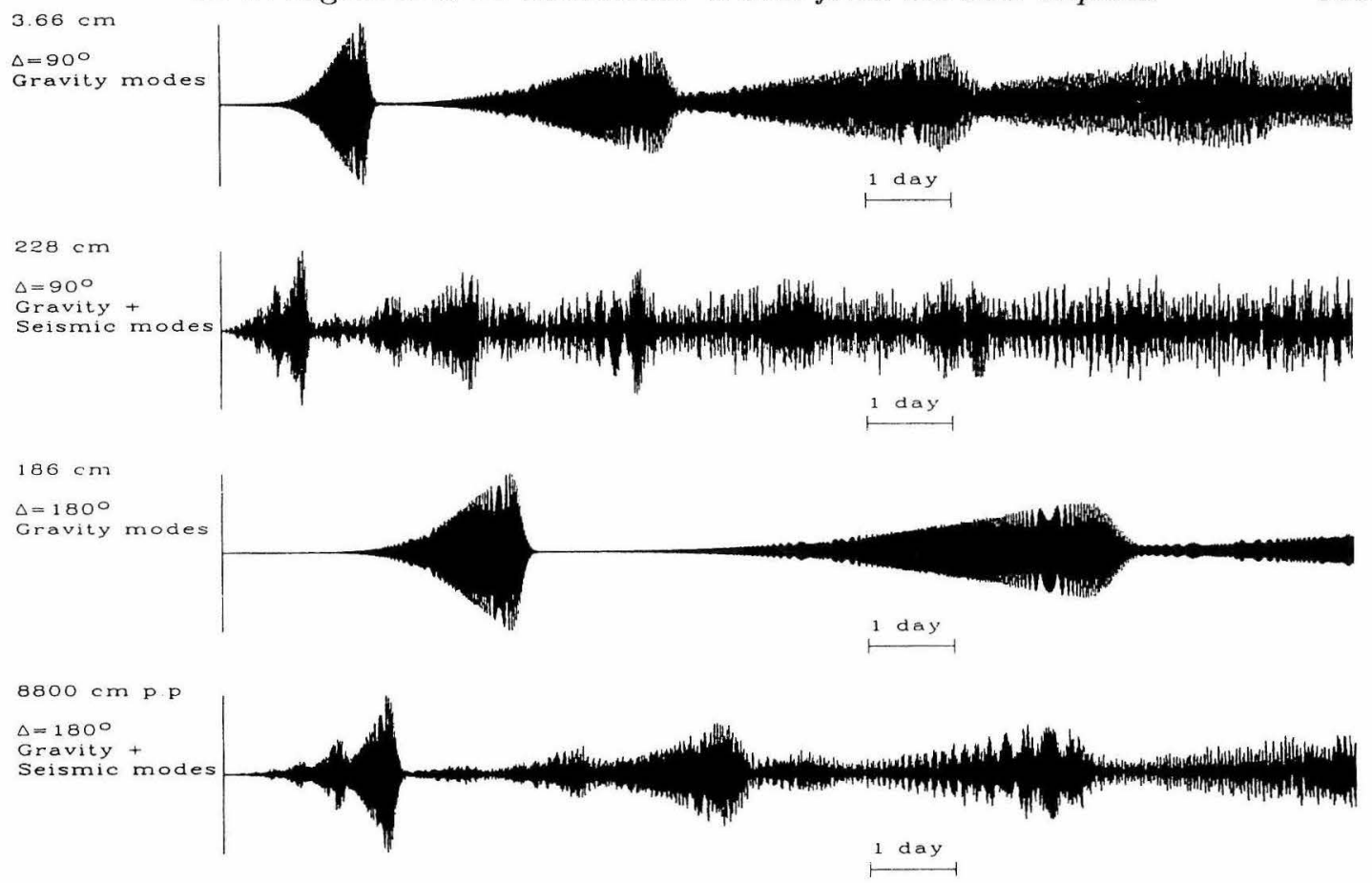

Figure 3. Vertical displacement as a function of time at locations $90^{\circ}$ and $180^{\circ}$ from the impact site. Displacement amplitude is given at left. Time increases to the right. The vertical line marks the time of impact. The first and third plots show surface gravity waves. The second and fourth plots show a superposition of surface gravity waves and acoustic waves. Reproduced from Lognonné et al. (1994).

According to Mosser et al. (1995), "Eight months after the impact of comet SL9 on Jupiter, it is still impossible to say whether the attempt to observe seismic waves excited by the impacts has been successfully conclusive."

\subsection{Direct ray}

Much of the interest in $p$-modes stems from a desire to learn about Jupiter's interior. The waves made up by superposition of high-frequency $p$-modes can be interpreted as seismic rays. Figure 4, reprinted from Hunten et al. (1994), shows the paths taken by rays launched at various angles with respect to the vertical. Only those launched nearly straight down can reach the plasma phase transition (PPT), the level below which hydrogen is a metal. Rays that are launched at other angles are refracted upward to the surface. The figure shows only the direct rays, which in seismological terms correspond to $p$-waves. The curves are labeled by the distance in $\mathrm{km}$ at which the ray re-surfaces.

Since all the rays start out at the same time but reach the surface at different times, the disturbance will appear to spread outward from the impact site. The position of the disturbance at various times after the impact is shown in Fig. 5, which is taken from Marley (1994). After 75 minutes, the disturbance radius is comparable to the radius of Jupiter-about $71,400 \mathrm{~km}$. The apparent speed is much faster than the speed measured by HST (Fig. 2), suggesting that the observed waves are not related to the direct $p$-wave. This conclusion is consistent with estimates by Marley (1994) that the wave amplitudes are greater than $0.1 \mathrm{~K}$ only for impacts larger than about $10^{28} \mathrm{ergs}$. This energy is larger than most estimates, so the direct $p$-wave is unlikely to be seen against the jovian weather background. 


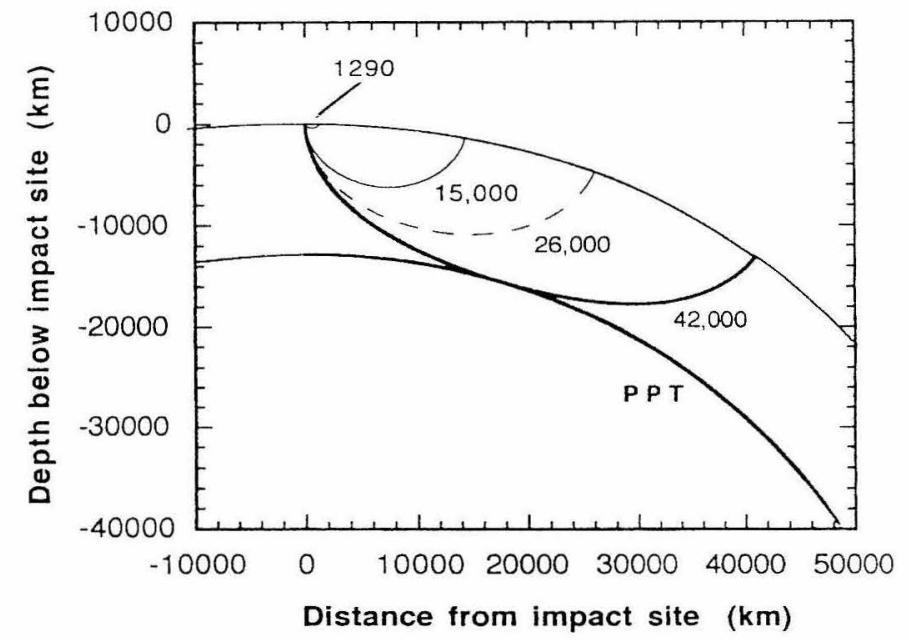

Figure 4. Paths of direct rays in Jupiter's interior. The rays differ in the initial angle with respect to the vertical, and are labeled by the horizontal distance traveled, in kilometers. PPT refers to the estimated location of the plasma phase transition. Reproduced from Hunten et al. (1994).

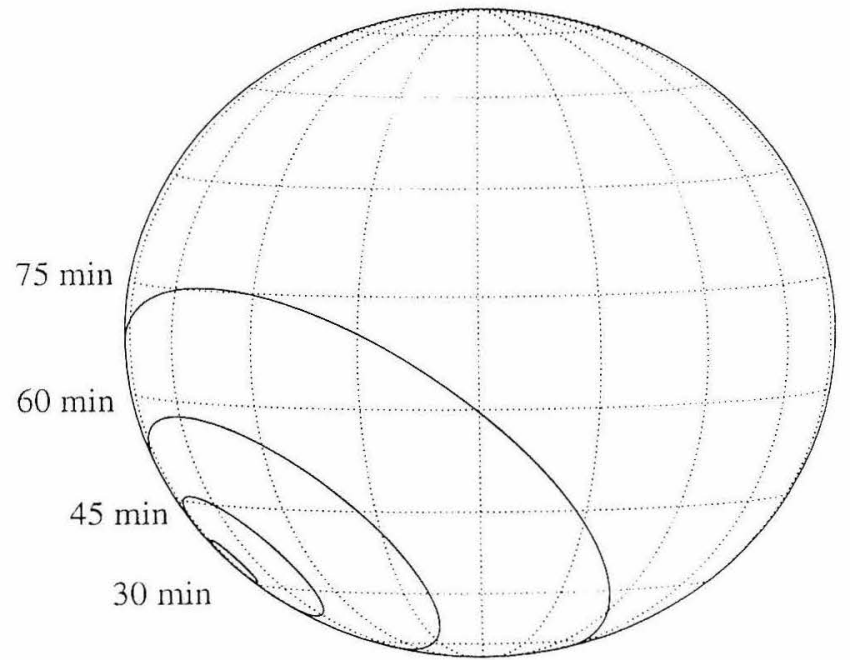

FIGURE 5. Intersection of the acoustic wave pulse (Fig. 4) with the surface, as a function of time from impact. The figure shows the appearance of the planet at various times if the amplitude were large. Reproduced from Marley (1994).

\subsection{Acoustic waveguide}

The speed of sound is proportional to $T^{1 / 2}$, where $T$ is temperature. The minimum temperature is found at the tropopause, where the pressure $P$ is $100 \mathrm{mb}$ and $T=110 \mathrm{~K}$. Figure 6 shows three profiles measured by the Voyager radio occultation experiment (Lindal et al. 1981). For Jupiter's hydrogen-helium atmosphere, the minimum speed of sound is $770 \mathrm{~m} \mathrm{~s}^{-1}$, which is too fast to match the observed speed (Fig. 2).

It is nevertheless useful to ask why sound waves were not observed. The tropopause acts as an acoustic waveguide: Sound waves can propagate horizontally over large distances because they do not lose energy through vertical propagation. Figure 7, reprinted from Collins et al. (1995), shows the normal modes of the acoustic waveguide. The dashed curve is the speed of sound, which varies with depth. The solid curves are the normal 


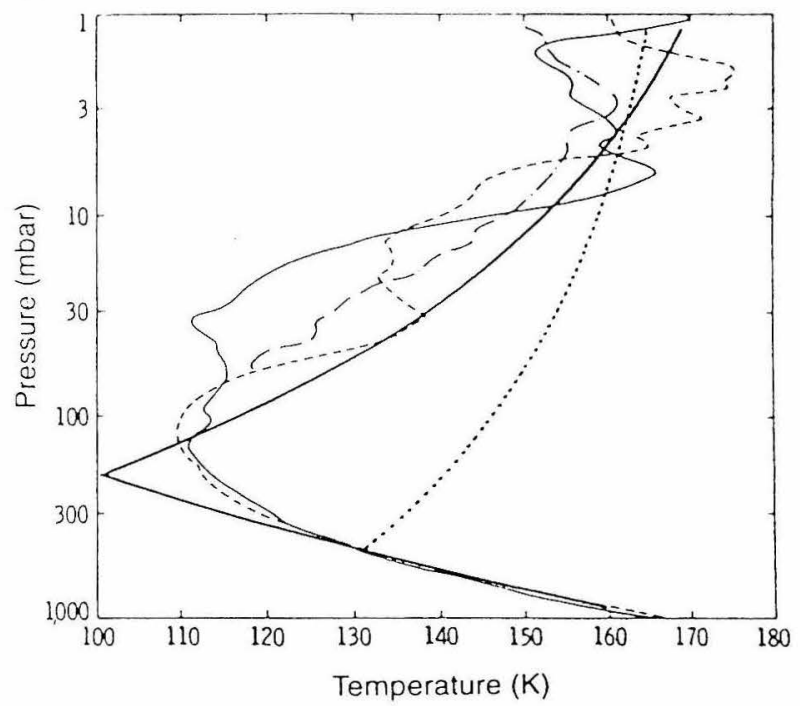

Figure 6. Temperature profiles in Jupiter's atmosphere. The light solid curve, the long dash curve, and the dot-dash curve are measured by the Voyager radio occultation experiment (Lindal et al. 1981). The heavy solid curve and the short dash curve are those used by Ingersoll \& Kanamori (1995). Reproduced from Ingersoll \& Kanamori (1995).

modes. The lowest mode (on the left) is a single peak centered on the temperature minimum; its amplitude falls off exponentially above and below this level. Both the phase and group speeds of this mode are near $770 \mathrm{~m} \mathrm{~s}^{-1}$. Higher modes have higher speeds. For a source near the tropopause most of the energy goes into the lowest mode. For a source at deeper levels the energy is distributed among all the modes, and the amplitude far from the source region is small.

Figure 8, reprinted from Ingersoll \& Kanamori (1995), shows the amplitude of the acoustic wave 1.5 hours after the impact for point sources at various pressure levels. The amplitude in this case is the Lagrangian temperature perturbation - the temperature change experienced by a parcel at the $126 \mathrm{mb}$ level. The source is a $10^{27} \mathrm{erg}$ pulse of heat at $r=0, t=0$. This is the kinetic energy of a solid sphere of ice of diameter $0.5 \mathrm{~km}$, travelling at the impact speed of the comet. This energy is consistent with current estimates for the larger fragments. As shown in Fig. 8, the response drops as the depth increases. For a source at 0.05 bar the amplitude of the response is $\pm 35 \mathrm{~K}$. For a source at 5 bar the amplitude is $\pm 0.65 \mathrm{~K}$, and for a source at 10 bar the amplitude is $\pm 0.20 \mathrm{~K}$. From the fact that sound waves were not seen, Ingersoll \& Kanamori (1995) conclude that the energy release was deep.

Another possibility is that the level of observation is high. A condensate is required to make the waves visible. Perhaps this condensate forms only above the $1 \mathrm{mb}$ level$100 \mathrm{~km}$ above the tropopause. There the amplitude of the lowest mode is small (Fig. 7), so the wave would be invisible to HST. Some combination of a source at low altitude and a condensate at high altitude is perhaps the most likely explanation for the absence of sound waves.

\section{6. $r$-modes and steady geostrophic motion}

Effects of rotation become important for low frequencies and long times. Lee \& Van Horn (1994) estimate the amplitude of inertial oscillations ( $r$-modes) in the interior of the planet, where buoyancy effects are negligible, and conclude that the oscillations would be unobservable unless the impact energy were greater than $10^{30}$ ergs. Ingersoll et al. (1994) 


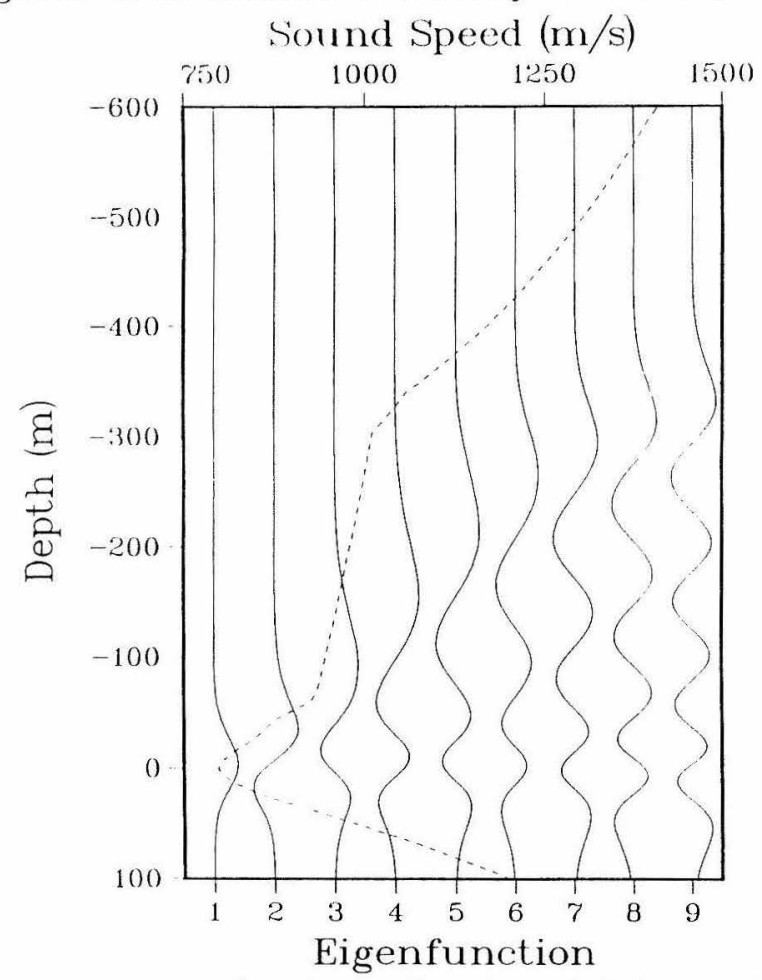

FIgURE 7. Sound speed (dashed curve) and eigenfunctions (solid curves) vs depth for acoustic waves trapped in the sound channel at the tropopause temperature minimum. Reproduced from Collins et al. (1995).

show that inertial oscillations become the largest mode in the atmosphere one day or more after the impact. They argue that the rings are inertia-gravity waves trapped in the water cloud, where buoyancy effects are important. As discussed in $\S 7.3$ of Gill (1982), the waves are dispersive. The short waves propagate away and leave the long waves behind. The latter are the inertial oscillations with frequency $f=2 \Omega \sin \phi$ where $\phi$ is latitude and $\Omega$ is the rotation rate of the planet. During the first 2.5 hours the effects of rotation are negligible, and the waves are indistinguishable from pure gravity waves unmodified by rotation.

Steady geostrophic flow is the limiting case of a low-frequency inertial mode (Greenspan 1968). On a rotating planet, such a flow can be excited by an initial transient. Gill (1982) calls this "The Rossby Adjustment Problem," and develops the theory for a single horizontal layer of fluid. Ingersoll et al. (1994) extend the theory to three dimensions, giving expressions for the steady circular vortex that remains after the inertia-gravity waves have propagated away. Harrington et al. (1994) obtained a steady vortex in their numerical experiments. The ultimate fate of the vortex depends on the ambient zonal flow. The HST observations do not distinguish between impact debris and impact-produced vortices. Hammel et al. (1995) do not report evidence of vortex-type motion at the impact sites, but another look at the images is warranted.

\section{Gravity Waves in the Water Cloud}

The key parameter for internal gravity waves ( $g$-modes) is $N$, the Brunt-Väisälä frequency of the atmosphere. This is the maximum frequency of a displaced parcel oscillating under its own buoyancy. $N^{2}$ is proportional to the difference between the lapse rate of 


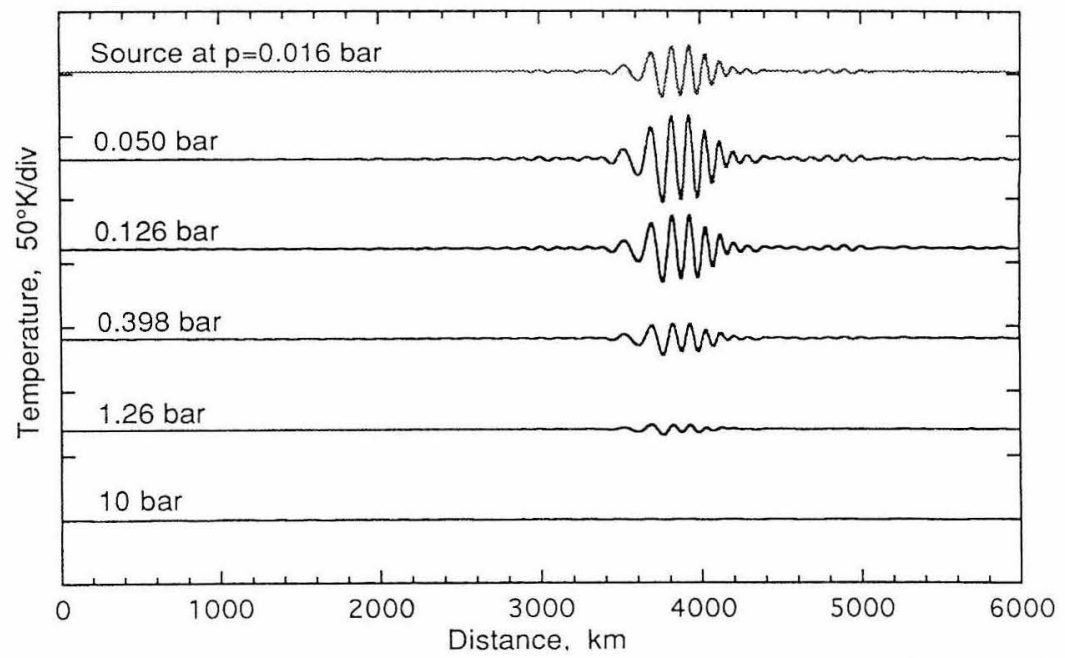

FIgure 8. Profiles of the acoustic wave amplitude as a function of distance, for sources at different altitudes. The time is 1.5 hours after the impact of a $10^{27}$ erg body. "Amplitude" refers to the temperature change experienced by a parcel at the $126 \mathrm{mb}$ level. The amplitude scale is $50 \mathrm{~K}$ per division, as shown at left. Reproduced from Ingersoll \& Kanamori (1995).

temperature and the adiabatic lapse rate. When temperature falls off more slowly than the adiabatic lapse rate, $N^{2}$ is positive. Then the atmosphere is said to be stable - the parcel executes sinusoidal oscillations about its equilibrium altitude.

Figure 9, reprinted from Ingersoll et al. (1994), shows the vertical profile $N(P)$ from $1 \mathrm{mb}$ to 10 bar. The thin wiggly line is computed from an average of the three Voyager radio occultation curves shown in Fig. 6. The data end at pressures slightly less than 1 bar, and the curve has been extended downward along a moist adiabat. The basic assumption is that moist convection makes the atmosphere stable, and that $N^{2}$ is proportional to the difference between a virtual moist adiabat and a dry adiabat. Observations in the Earth's tropics (Emanuel 1994, pp. 472-491) show that the mean density structure is close to that of a virtual moist adiabat, which is the density of a rising parcel undergoing latent heat release and change of molecular weight as the condensate falls out. This mean density structure is maintained by convection in isolated "cores" of cumulonimbus clouds that occupy only $0.1 \%$ of the area (Riehl \& Malkus 1958; Palmén \& Newton 1969). Outside the cores the atmosphere is unsaturated - the relative humidity is less than one. So a wave that does not cause the atmosphere to saturate will propagate dry adiabatically. Thus the atmosphere is neutral with respect to moist convection but is stable with respect to large-scale waves.

In Fig. 9 the water cloud base is at 5 bar. This is the saturation point in a "solar composition" atmosphere one where the $\mathrm{O} / \mathrm{H}$ ratio of Jupiter's interior is the same as that on the Sun (Gautier \& Owen 1989). Since water is the main oxygen-bearing molecule, the $\mathrm{O} / \mathrm{H}$ ratio sets the water abundance. $N^{2}$ is large at cloud base and falls abruptly to zero below it. Ingersoll \& Kanamori (1995) use simple analytic functions fitted to the Voyager profile in the stratosphere and the moist adiabat in the water cloud, for $\mathrm{O} / \mathrm{H}$ ratios up to 10 times solar. The fitted function for solar $\mathrm{O} / \mathrm{H}$ is shown in Fig. 9. If we define $e_{H_{2} \mathrm{O}}$ as the $\mathrm{O} / \mathrm{H}$ abundance on Jupiter divided by the $\mathrm{O} / \mathrm{H}$ abundance on the Sun, then "solar" composition corresponds to $e_{\mathrm{H}_{2} \mathrm{O}}=1$.

Energy from the impacts tends to excite two kinds of internal gravity waves, corresponding to the two regions with large $N^{2}$ in Fig. 9. The amplitude of the stratospheric gravity wave (SGW) compared to that of the tropospheric gravity wave (TGW) depends 


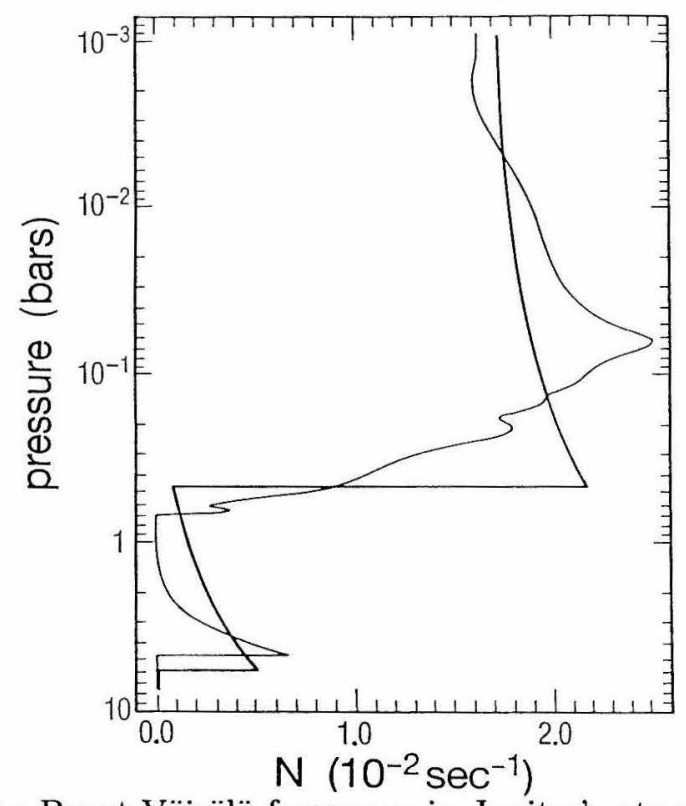

Figure 9. Profiles of the Brunt-Väisälä frequency in Jupiter's atmosphere. The light wiggly curve is derived from the Voyager radio occultation data (Fig. 6), with a moist adiabatic extrapolation below the 1 bar level. The heavy smooth curve is derived from an analytic fit to the temperature data. Reproduced from Ingersoll et al. (1994).

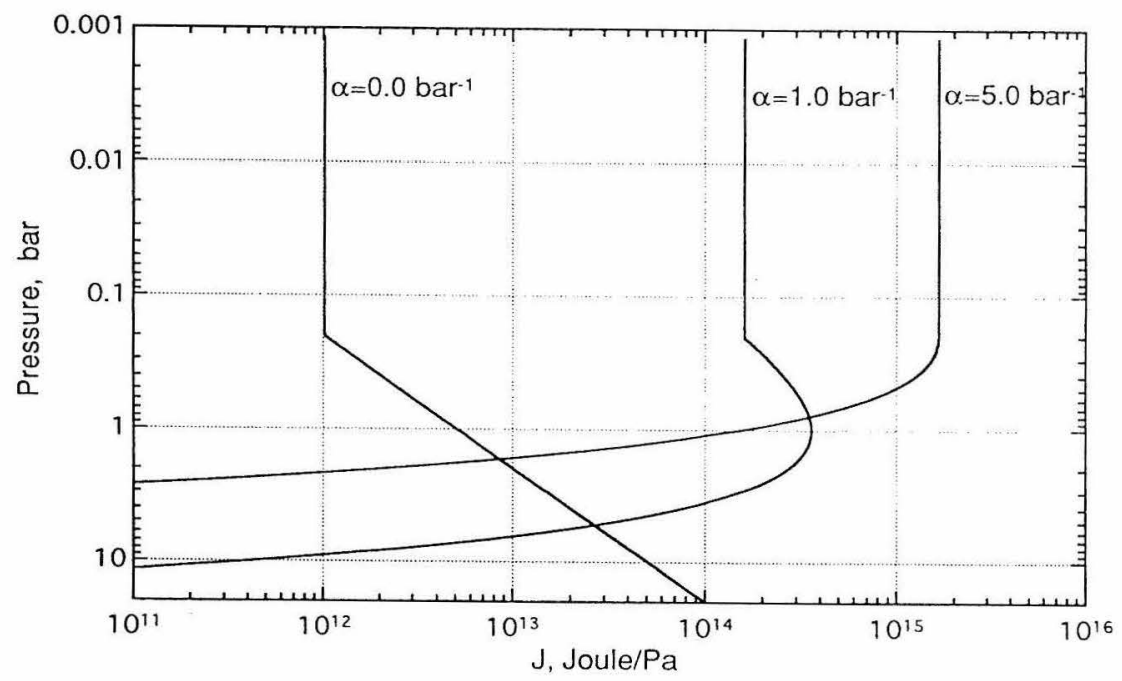

Figure 10. Vertical profiles of heating used by Ingersoll \& Kanamori (1995) in their calculation of inertia-gravity waves from the SL9 impacts. The ordinate is the energy density in $\mathrm{J} \mathrm{Pa}^{-1}$, and the integral of each curve with respect to pressure is $10^{27} \mathrm{erg}$. Reproduced from Ingersoll \& Kanamori (1995).

on the vertical distribution of heating during the impact. Figure 10 shows three heating profiles used by Ingersoll \& Kanamori (1995). The parameter $\alpha$ has units of inverse pressure. Large $\alpha$ corresponds to heating at small pressure, and vice versa. Heating is zero below cloud base, which in this case is at 20 bar, corresponding to 10 times the solar abundance of water. The units of heating are $\mathrm{J} \mathrm{Pa}^{-1}$, and the integral of each curve with respect to pressure is $10^{27} \mathrm{erg}$. This is the total impact energy for this simulation. 


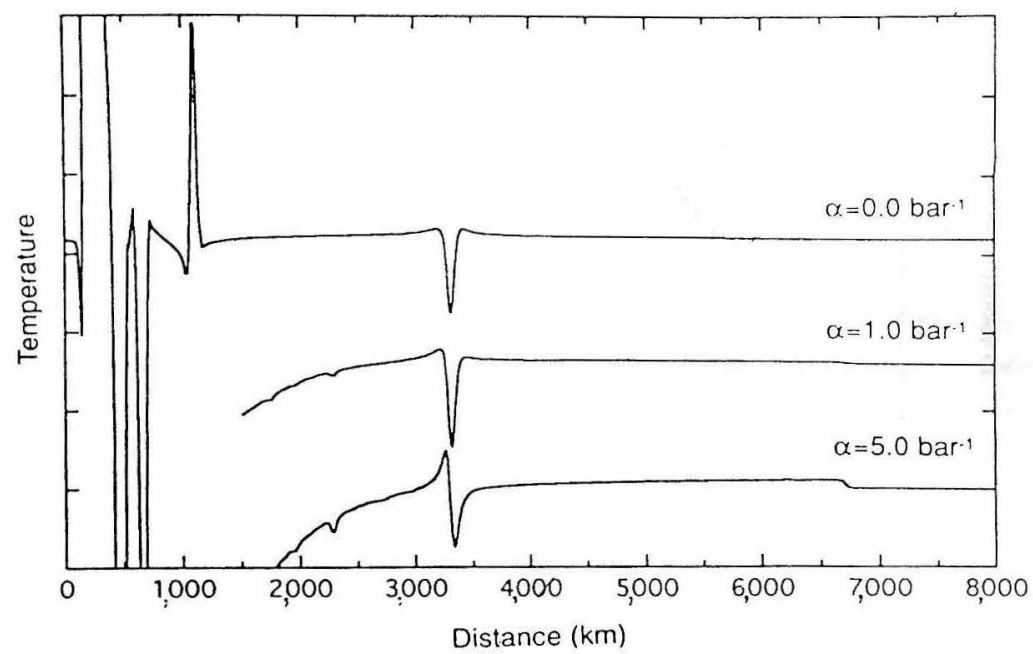

Figure 11. Profiles of gravity wave amplitudes as a function of distance, for the three different heating profiles of Fig. 10. "Amplitude" is the temperature change experienced by a parcel at the $45 \mathrm{mb}$ level. The time is 2 hours after the impact of a $10^{27}$ erg body. Units are $1 \mathrm{~K}$ per division on the vertical axis. The pulse at $3300 \mathrm{~km}$ is the fundamental gravity wave mode in the water cloud. The pulse at $1100 \mathrm{~km}$ is the next higher mode. The bump at $6700 \mathrm{~km}$ in the $\alpha$ $=5 \mathrm{bar}^{-1}$ curve (heating at high altitude) is the gravity wave in the stratosphere. Reproduced from Ingersoll \& Kanamori (1995).

Figure 11, which is also reprinted from Ingersoll \& Kanamori (1995), shows three snapshots of internal gravity waves corresponding to the three heating profiles of Fig. 10. The ordinate is the Lagrangian temperature perturbation at the $45 \mathrm{mb}$ level. The abscissa is radial distance from the impact point, and the time is two hours (7200 s) after the impacts. The model uses the analytic temperature profile represented by the heavy solid line in Fig. 6. The extension to the base of the water cloud at $P=20$ bars is shown in Fig. 12. This model, with $e_{\mathrm{H}_{2} \mathrm{O}}=10$, is the one that matches the $450 \mathrm{~m} \mathrm{~s}^{-1}$ speed of the rings observed in the HST images. As shown in Ingersoll et al. (1994), the speed varies as the square root of the water abundance and little else. For $e_{\mathrm{H}_{2} \mathrm{O}}=1$ the speed is $130 \mathrm{~m} \mathrm{~s}^{-1}$.

In Fig. 11, the pulse at $r=3300 \mathrm{~km}$ is the TGW travelling at $450 \mathrm{~m} \mathrm{~s}^{-1}$. Although the wave has most of its energy in the water cloud, it leaks energy into the stratosphere where we can observe it with HST. As in Fig. 2 of Ingersoll et al. (1994), the disturbance amplitude is considerably larger at $45 \mathrm{mb}$ than it is at 1 bar or at the $\mathrm{NH}_{3}$ cloud tops near $500 \mathrm{mb}$. As pointed out by Achterberg \& Ingersoll (1989), the disturbance grows in amplitude and oscillates with height in the stratosphere. Allison (1990) showed that the vertical wavelength roughly matches that observed in the radio occultation profiles (Fig. 6), provided the water abundance is $2-3$ times solar.

The response function also increases with height in the stratosphere: For a given input energy the amplitude of the TGW is greater when the source is at high altitude than when the source is at low altitude. The same is true of atmospheric tides on Earth, which are internal gravity waves forced by solar heating: Absorption of small amounts of sunlight in the mesosphere by water vapor and ozone makes a large contribution to the tidal pressure oscillation at the ground (Chapman \& Lindzen 1970).

In Fig. 11 the disturbance at $r=6700 \mathrm{~km}$ is the leading edge of the SGW. Its speed is $930 \mathrm{~m} \mathrm{~s}^{-1}$ and is very hard to change. We have run cases with $e_{\mathrm{H}_{2} \mathrm{O}}$ ranging from 0.5 to 10 , and we have tried putting all the heat at one level, varying the pressure of the level 


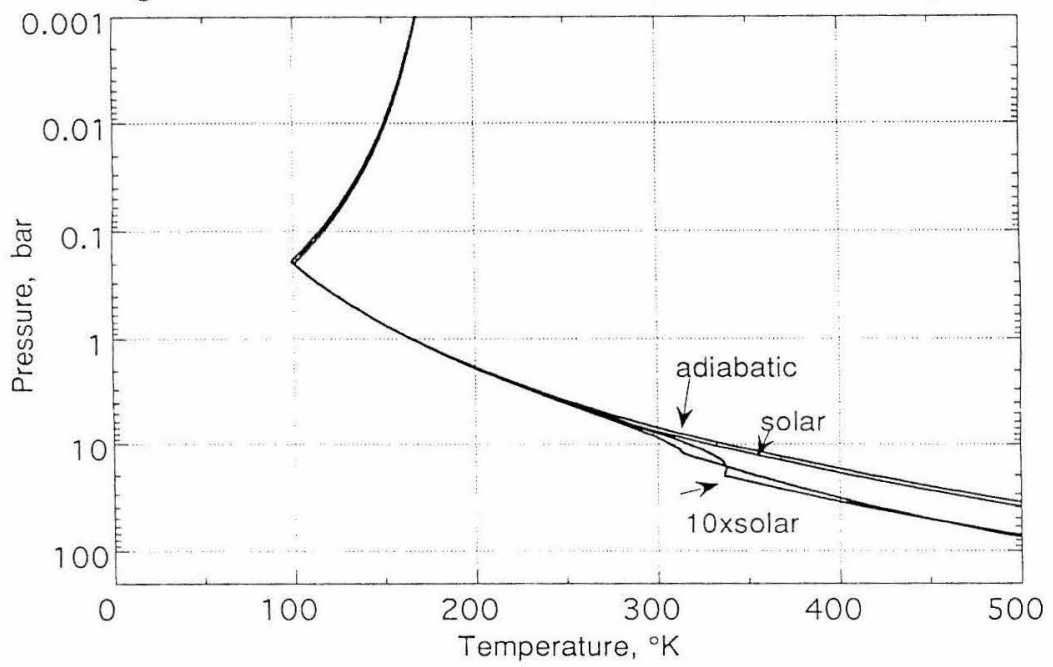

Figure 12. Profiles of virtual temperature $T m_{d r y} / m$ for the no-water (adiabatic), solar, $5 \times$ solar, and $10 \times$ solar cases used by Ingersoll and Kanamori (1995) in their gravity wave calculations, where $m$ is the molecular mass of the mixture and $m_{d r y}$ is the molecular mass of the atmosphere without condensate.

from $10 \mathrm{mb}$ to 10 bar. We have increased the pressure at the tropopause by a factor of three (Fig. 6, dashed line), and we have varied $T_{\infty}$-the temperature at the top of the stratosphere. The speed of the SGW depends only on $T_{\infty}$, varying as $T_{\infty}^{1 / 2}$. It does not match the $450 \mathrm{~m} \mathrm{~s}^{-1}$ observed speed for any reasonable profile. And in all cases the amplitude of the SGW is less than that of the TGW. We conclude that the observed waves are TGWs, not SGWs.

This conclusion is supported by the model described in Harrington et al. (1994). Running their model with 40 vertical layers gives results almost identical to those in Fig. 11. With 40 vertical layers the numerical model successfully mimics the dissipation of wave energy propagating up from the troposphere (Lindzen et al. 1968). With just 5 vertical layers the model gives spurious refections from the upper layer, which reduces the speed of the SGW.

The disturbance at $r=1100 \mathrm{~km}$ in Fig. 11 is the second mode of the tropospheric waveguide. For this temperature profile (Figs. 6 and 12), the mode travels at $150 \mathrm{~m} \mathrm{~s}^{-1}$, one-third the speed of the first mode. As described in Ingersoll et al. (1994), the first mode spans one quarter-cycle and the second mode spans three quarter-cycles from cloud base to tropopause. The two modes might be related to the outer and inner rings, although the ratio of the two speeds (3 to 1 in the model) does not exactly match the observed ratio (about 2.15 to 1 ).

\section{Implications and conclusions}

Other inferences about jovian water tend to support $e_{H_{2} O} \geq 1$, but there are major uncertainties. Bjoraker et al. (1986) looked at both the high-resolution spectra taken from the Kuiper Airborne Observatory and the lower-resolution spectra taken by the Voyager IRIS. They concluded that $e_{\mathrm{H}_{2} \mathrm{O}} \sim 1 / 50$. Analysing just the Voyager data, Carlson et al. (1992) conclude $e_{\mathrm{H}_{2} \mathrm{O}} \geq 1$. They argue that $e_{\mathrm{H}_{2} \mathrm{O}}=10$ fits the data equally well (see their Fig. 9). The high-resolution data are best for isolating individual spectral lines, particularly the weak ones. With Voyager IRIS one is limited to strong: 
lines only. However the main difference between the two models concerns assumptions made about scattering in the clouds.

Voyager and Earth-based observations (Gautier \& Owen 1989) give $e_{\mathrm{CH}_{4}} \sim e_{\mathrm{NH}_{3}} \sim$ 2 and $e_{H e} \sim 0.7$. Analysis of the gravitational harmonics of Jupiter (Hubbard 1989) suggests at least a 5-fold enrichment of heavy elements like O, C, and N. This enrichment drops by a factor of two if Jupiter has a subadiabatic (radiative) zone in the 1-40 kbar pressure range, as suggested by Guillot et al. (1994).

The Galileo spacecraft encountered Jupiter on December 7, 1995. The probe sent back useful data from above cloud tops down to the 20 bar level. Preliminary results suggest that water is present at less than solar abundance. The mass spectrometer found less water than the solar value, and the temperature profile measured by the atmospheric structure instrument was closer to a dry adiabat than to a moist adiabat. Also, the nephelometer found almost no cloud particles at the level of the purported water cloud, and the net flux radiometer found low opacity in the 5-micron band where water vapor is a dominant absorber.

Thus the preliminary Galileo results do not support the inferences made from studying the SL9 waves. There are a number of possibilities. First, the water vapor abundance may vary from place to place (the SL9 fragments entered the atmosphere at a latitude of $-44^{\circ}$, and the Galileo probe entered at $6.5^{\circ}$ ). Second, the identification of the ring as a wave in the jovian water cloud may be flawed. And third, the Galileo results may change after further calibration of the instruments.

If there is a deep stable layer extending down below 10 bars over most of the planet, then our best guess is that this layer is maintained by moist convection, with $e_{\mathrm{H}_{2} \mathrm{O} \sim}$ 10. Such a result has important implications regarding the origin of Jupiter, its internal history, and the chemistry and meteorology of its current atmosphere.

Ingersoll acknowledges support from NASA's Planetary Atmospheres Program, grant NAGW-1956, and from NASA's Space Telescope Science Institute; Kanamori acknowledges support from NSF grant EAR-92 18809.

\section{REFERENCES}

Achterberg, R. K. \& Ingersoll, A. P. 1989 A normal-mode approach to jovian atmospheric dynamics. J. Atmos. Sci. 46, 2448-2462.

Allison, M. 1990 Planetary waves in Jupiter's equatorial atmosphere. Icarus 83, 282-307.

Bjoraker, G. L., Larson, H. P. \& Kunde, V. G. 1986 The gas composition of Jupiter derived from 5- $\mu \mathrm{m}$ airborne spectroscopic observations. Icarus 66, 579-609.

CArlson, B. E., LACIS, A. A. \& Rossow, W. B. 1992 The abundance and distribution of water vapor in the jovian troposphere as inferred from Voyager IRIS observations. Astrophys. J. 388, 648-668.

Chapman, S. \& Lindzen, R. S. 1970 Atmospheric Tides. Gordon and Breach Science Publishers.

Collins, M., McDonald, B. E., Kupperman, W. A. \& Siegmann, W. L. 1995 Jovian acoustics and comet Shoemaker-Levy 9. J. Acoust. Soc. Amer. 97, 2147-2158.

DEming, D. 1994 Prospects for jovian seismological observations following the impact of comet Shoemaker-Levy 9. Geophys. Res. Lett. 21, 1095-1098.

Emanuel, K. A. 1994 Atmospheric Convection, Oxford University Press.

GaUtier, D. \& OWEN, T. 1989 The composition of outer planet atmospheres. In Origin and Evolution of Planetary and Satellite Atmospheres (ed. S. K. Atreya, J. B. Pollack \& M. S. Matthews), pp. 487-512. University of Arizona Press.

Gill, A. E. 1982 Atmosphere-Ocean Dynamics, Academic Press. 
Goldreich, P. \& Kumar, P. 1990 Wave generation by turbulent convection. Astrophys. J. 363, 694-704.

Gough, D. O. 1994 Seismic consequences of the Shoemaker-Levy impact. Mon. Not. R. Astron. Soc. 269, L17-L20.

Greenspan, H. P. 1968 The Theory of Rotating Fluids, Cambridge University Press.

Guillot, T., Chabrier, G., Morel, P. \& Gautier, D. 1994 Nonadiabatic models of Jupiter and Saturn. Icarus 112, 354-357.

Hammel, H. B., Beebe, R. F., Ingersoll, A. P., Orton, G. S., Mills, J. R., Simon, A. A., Chodas, P., Clarke, J. T., De Jong, E., Dowling, T. E., Harrington, J., Huber, L. F., Karkoschka, E., Santori, C. M., Toigo, A., Yeomans, D. \& West, R. A. 1995 HST imaging of atmospheric phenomena created by the impact of comet ShoemakerLevy 9. Science 267, 1288-1296.

Harrington, J., LeBeau, R. P., Backes, K. A. \& Dowling, T. E. 1994 Dynamic response of Jupiter's atmosphere to the impact of comet Shoemaker-Levy 9. Nature 368, 525-527.

HuBbard, W. B. 1989 Structure and composition of giant planet interiors. In Origin and Evolution of Planetary and Satellite Atmospheres (ed. S. K. Atreya, J. B. Pollack \& M. S. Matthews), pp. 539-563. University of Arizona Press.

Hunten, D. M., Hoffmann, W. F. \& Sprague, A. L. 1994 Jovian seismic waves and their detection. Geophys. Res. Lett. 21, 1091-1094.

Ingersoll, A. P., Kanamori, H. \& Dowling, T. E. 1994 Atmospheric gravity waves from the impact of comet Shoemaker-Levy 9 with Jupiter. Geophys. Res. Lett. 21, 1083-1086.

Ingersoll, A. P. \& KANAmori, H. 1995 Waves from the collisions of comet Shoemaker-Levy 9 with Jupiter. Nature 374, 706-708.

Kanamori, H. 1993 Excitation of jovian normal modes by an impact source. Geophys. Res. Lett. 20, 2921-2924.

LEE, U. \& VAN HORN, H. M. 1994 Global oscillation amplitudes excited by the Jupiter-comet collision. Astrophys. J. 428, L41-L44.

Lindal, G. F., Wood, G. E., Levy, G. S., Anderson, J. D., Sweetnam, D. N., Hotz, H. B., Buckles, B. J., Holmes, D. P., Doms, P. E., Eshleman, V. R., Tyler, G. L. \& Croft, T. A. 1981 The atmosphere of Jupiter: An analysis of the Voyager radio occultation measurements. J. Geophys. Res. 86, 8721-8727.

Lindzen, R. S., Batten, E. S. \& KIM, J. W. 1968 Oscillations in atmospheres with tops. Mon. Wea. Rev. 96, 133-140.

Lognonné, P., Mosser, B. \& Dahlen, F. A. 1994 Excitation of jovian seismic waves by the Shoemaker-Levy 9 cometary impact. Icarus 110, 180-195.

MARLEY, M. S. 1994 Seismological consequences of the collision of comet Shoemaker-Levy/9 with Jupiter. Astrophys. J. 427, L63-L66.

McGregor, P. J., Nicholson, P. D. \& Allen, M. G. 1995 CASPIR observations of the collision of comet Shoemaker-Levy 9 with Jupiter. Icarus, in press.

Mosser, B., Galdemard, P., Jouan, R., Lagage, P., Masse, P., Pantin, E., Sauvage, M., Lognonné, P., Gautier, D., Drossart, P., Merlin, P., Sibille, F., Vauglin, I., Billebaud, F., Livengood, T., Käufi, H. U., Marley, M., Hultgren, M., Nordh, L., Olofsson, G., Ulla, A., Belmonte, J. A., Regulo, C., Roca-Cortez, C., Selby, M., Rodriguez-EspinosA, J. M. \& VIDAL, I. 1995 Seismic studies of Jupiter at the time of SL-9 impacts. In European SL-9/Jupiter Workshop, Garching, 13-15 February 1995 (eds. R. M. West \& H. Boehnhardt), pp. 397-402. European Southern Observatory Conference and Workshop Proceedings.

Palmén, E. \& Newton, C. W. 1969 Atmospheric Circulation Systems, Academic Press.

Pankine, A. A. \& Ingersoll, A. P. 1995 Ejecta patterns of the impacts of comet ShoemakerLevy 9. Bull. Amer. Astron. Soc. 27, 76.

Riehl, H. \& Malkus, J. S. 1958 On the heat balance in the equatorial trough zone. Geophysica (Helsinki) 6, 503-537. 
West, R. A., Karkoschka, E., Friedson, A. J., Seymour, M., Baines, K. H. \& Hammel, H B. 1995 Impact debris particles in Jupiter's stratosphere. Science 267, 1296-1301.

ZAHnLE, K. 1995 Dynamics and chemistry of SL9 plumes. This volume.

Zel'dovich, YA. B. \& RaIZer, YU. P. 1967 Physics of Shock Waves and High-Temperature Hydrodynamic Phenomena, Volume II, Academic Press.

Zhang, H. \& INGersoll, A. P. 1995 Rings in HST images of SL9 collision with Jupiter: Waves or advection? Bull. Amer. Astron. Soc. 27, 76. 